\title{
Functional cardiac MRI in preterm and term newborns
}

\author{
Alan M Groves, ${ }^{1}$ Gaia Chiesa, ${ }^{1}$ Giuliana Durighel, ${ }^{1}$ Stephen T Goldring, ${ }^{1}$ Julie A Fitzpatrick, \\ Sergio Uribe, ${ }^{2,3}$ Reza Razavi, ${ }^{2}$ Jo V Hajnal, ${ }^{1}$ A David Edwards ${ }^{1}$
}

IInstitute of Clinical Sciences, Imperial College and MRC Clinical Sciences Centre, Hammersmith Hospital, London, UK

${ }^{2}$ Division of Imaging Sciences, British Heart Foundation (BHF) National Centre, National Institute for Health Research (NIHR) Comprehensive Biomedical Research Centre, Guy's and St Thomas' National Health Service (NHS) Foundation Trust, King's College London, London, UK ${ }^{3}$ Radiology Department and Center for Biomedical Imaging, Pontificia Universidad Catolica de Chile, Santiago, Chile

\section{Correspondence to}

Dr Alan Groves, Department of Paediatrics, Hammersmith House, Hammersmith Hospital, Du Cane Road, London W12 ONN, UK;

alan.groves@imperial.ac.uk

Accepted 13 September 2010 Published Online First 21 October 2010

\begin{abstract}
Objective To use cardiac MRI techniques to assess ventricular function and systemic perfusion in preterm and term newborns, to compare techniques to echocardiographic methods, and to obtain initial reference data.

Design Observational magnetic resonance and echocardiographic imaging study.

Setting Neonatal Unit, Queen Charlotte's and Chelsea Hospital, London, UK.
\end{abstract}

Patients 108 newborn infants with median birth weight 1627 (580-4140) g, gestation 32 (25-42) weeks.

Results Mean (SD) flow volumes assessed by phase contrast (PC) imaging in 28 stable infants were left ventricular output (LVO) 222 (46), right ventricular output (RVO) 219 (47), superior vena cava (SVC) 95 (27) and descending aorta (DAo) 126 (32) $\mathrm{ml} / \mathrm{kg} / \mathrm{min}$, with flow being higher at lower gestational age. Limits of agreement for repeated PC assessment of flow were LVO \pm 50.2 , RVO \pm 55.5 , SVC \pm 20.9 and DAo $\pm 26.2 \mathrm{ml} / \mathrm{kg} / \mathrm{min}$. Mean (SD) LVO in 75 stable infants from three-dimensional models were 245 (47) ml/ $\mathrm{kg} / \mathrm{min}$, with limits of agreement $\pm 58.3 \mathrm{ml} / \mathrm{kg} / \mathrm{min}$. Limits of agreement for repeated echocardiographic assessment of LVO were $\pm 108.9 \mathrm{ml} / \mathrm{kg} / \mathrm{min}$.

Conclusions Detailed magnetic resonance assessments of cardiac function and systemic perfusion are feasible in newborn infants, and provide more complete data with greater reproducibility than existing echocardiographic methods. Functional cardiac MRI could prove to be a useful research technique to study small numbers of newborn infants in specialist centres; providing insights into the pathophysiology of circulatory failure; acting as an outcome measure in clinical trials of inotropic intervention and so guiding clinical practice in the wider neonatal community.

More than 50000 infants are born prematurely in the UK annually, with a socioeconomic burden of $>£ 3$ billion. ${ }^{1}$ Circulatory failure is responsible for a high proportion of neonatal mortality, ${ }^{2}$ particularly when associated with sepsis, and is implicated in the pathogenesis of neonatal cerebral ${ }^{3}$ and pulmonary injury. ${ }^{4}$

Cardiovascular physiology in the preterm infant is poorly understood and may differ significantly from older children; as there is significant disparity in adrenergic receptor number, distribution and sensitivity. ${ }^{5}$ The effect of inotropic intervention in the preterm neonate is not well-described, leading to a tenfold variation in inotropic intervention between neonatal units. ${ }^{7}$ Research to enhance understanding of neonatal circulatory failure is a priority. 58

Functional echocardiographic techniques have been employed to study the neonatal circulation, however, currently available methods are unable to detect changes in volume of blood flow of $<30-40 \%,{ }^{9-11}$ and cannot reliably assess ejection fraction (EF) due to asymmetric left ventricular contours. ${ }^{12}$ Cardiac magnetic resonance (CMR) has become the gold standard for functional assessment in adults, ${ }^{13}$ giving significantly improved reproducibility over echocardiography, ${ }^{14}$ facilitating reduced sample sizes for clinical trials of circulatory intervention. ${ }^{15}$

We have installed a magnetic resonance scanner within our neonatal intensive care unit (NICU) to allow us to study extremely preterm infants while maintaining circulatory, respiratory and thermal stability. ${ }^{16}$ We have previously acquired basic cine CMR images in newborns without sedation or anaesthesia, ${ }^{17}$ but techniques had not previously been optimised or validated. The aim of this study was to utilise optimised CMR techniques to assess cardiac filling, cardiac output and systemic perfusion in a large cohort of preterm and term newborns, to test the hypothesis that neonatal CMR measures would provide greater detail and reproducibility than existing echocardiographic techniques, and to obtain initial reference data.

\section{SUBJECTS AND METHODS Subjects}

We studied infants who were inpatients at Queen Charlotte's and Chelsea Hospital. MR imaging was approved by the Hammersmith Hospitals Trust Research Ethics Committee, and written parental consent was obtained for all studies. Infants were scanned solely for cardiac imaging research.

All infants were stable at the time of imaging, whether receiving nasal continuous positive airway pressure via an MR compatible system, ${ }^{16}$ low flow supplemental oxygen or no respiratory support. All infants were tolerating full enteral feeds.

\section{Cardiovascular magnetic resonance}

Scans were performed with a Philips 3-Tesla (Best, Netherlands) MR scanner. A paediatrician was present throughout. Infants were fed and allowed to fall into natural sleep without sedation or anaesthesia, then laid in an MRI-compatible cradle with ear protection, pulse oximetry and vector ECG monitoring. ${ }^{17}$ A Flex-M two-channel surface receiver coil was placed on either side of the chest wall. All sequences were acquired freebreathing, no respiratory compensation techniques were used.

For all examinations, specific absorption rate (SAR) levels were within agreed safe limits (local SAR $<10 \mathrm{~W} / \mathrm{kg}$, whole body SAR $<0.8 \mathrm{~W} / \mathrm{kg}$ ). Entire imaging examination took 45-60 min. 


\section{Phase contrast imaging}

After initial survey for determination of cardiac position, steady-state free procession (SSFP) short axis and vertical and horizontal long axis views were obtained from initial axial scan through the heart (figure 1 with video links). Aortic and pulmonary outflow tracts were imaged using two orthogonal SSFP sequences. Left and right ventricular output (LVO and RVO) were assessed using through-plane phase contrast (PC) techniques with slice positioned immediately distal to the aortic and pulmonary valves. ${ }^{18}$ Since patency of the ductus arteriosus or foramen ovale prevent LVO and RVO from indicating systemic perfusion in the newborn, volumes of flow in the superior vena cava (SVC) and descending aorta (DAo) were also assessed as markers of upper and lower body perfusion. ${ }^{10} 11$ Axial stacks of T1-weighted spin echo images were taken and multiplanar reformatted into sagittal and coronal planes showing the SVC and DAo, with flow assessed by $\mathrm{PC}$ at the level of the pulmonary bifurcation and diaphragm, respectively. Acquisition parameters for PC imaging were repetition time $8.4 \mathrm{~ms}$, echo time $4.9 \mathrm{~ms}$, flip angle $10^{\circ}$, slice thickness $4 \mathrm{~mm}$, acquired voxel size $1.2 \mathrm{~mm}, 20$ phases/cycle, scan duration $57 \mathrm{~s}$. Velocity encoding was set at the minimum level to prevent aliasing, generally $100 \mathrm{~cm} / \mathrm{s}$ for LVO, RVO and DAo flow, $60 \mathrm{~cm} / \mathrm{s}$ for SVC flow.

\section{PC analysis and validation}

All PC sequences were analysed using an in-built workstation (Philips ViewForum). Automated vessel edge detection was used for pulmonary and descending aortic contours, with manual adjustment where necessary. Aortic contour was traced using a visually applied ellipse function. SVC contour was traced manually. PC sequences were externally validated against a flow phantom with $8 \mathrm{~mm}$ internal diameter, which approximates the size of the newborn aorta, ${ }^{19}$ with non-pulsatile flow rates of 0-1200 $\mathrm{ml} / \mathrm{min}$, and using automated vessel edge detection.

\section{Cine imaging}

Cine imaging was performed using SSFP. From vertical and horizontal long axis views obtained as above, a contiguous stack of short axis images (5-7 slices) was obtained to cover the left ventricle from atrioventricular (A-V) valve to cardiac apex. Acquisition parameters for cine imaging were repetition time $4.1 \mathrm{~ms}$, echo time $2.1 \mathrm{~ms}$, flip angle $45^{\circ}$, slice thickness 3-5 mm, acquired voxel size $1.5 \mathrm{~mm}, 32$ phases/cycle, scan duration 29 s/slice.

\section{Cine image analysis}

Cine images were analysed using semiautomated segmentation software (CMR Tools; CVIS, London, UK). Left and right ventricular end-diastolic (EDV) and end-systolic (ESV) volumes, stroke volume and EF were calculated by tracing the endocardial

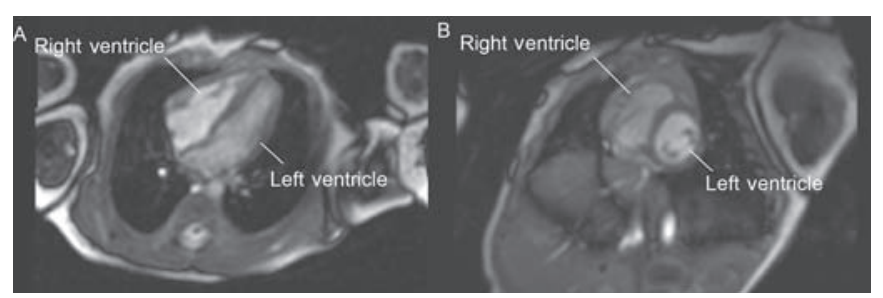

Figure 1 Four chamber $(A)$ and short axis $(B)$ views obtained with balanced fast field echo sequences in a newborn infant (view also video links at http://adc.bmj.com). border at end-systole and end-diastole at each level of the short axis stack. The first image after the $\mathrm{R}$ wave was considered to be end-diastole. The end-systolic phase was determined as the slice with the smallest ventricular cavity. Volumes of the papillary muscles and moderator band were excluded from the ventricular volumes. The A-V valve planes were tracked through the cardiac cycle from two chamber and four chamber views. Measures of signal to noise ratio (SNR) in the myocardium and blood; and contrast to noise ratio (CNR) between myocardium and blood were taken by standard means. ${ }^{20}$

\section{Echocardiographic imaging}

CMR was also used to study a sub-cohort of infants who had cardiac structure and function assessed by echocardiography within 24 h of CMR. Echocardiography scans were performed by a neonatologist with 10 years' echo experience (AMG) using an Antares system (Siemens, Mountain View, California, USA) with a $10 \mathrm{MHz}$ linear array transducer. Cardiac structural abnormalities, significant intra- and extra-cardiac shunts and $A-V$ valve regurgitation were excluded. LVO was quantified to allow comparison with CMR. Left ventricular outflow tract diameter was assessed at the level of the aortic valve on 2D imaging at end-systole, aortic blood flow velocity was assessed using pulsed wave Doppler placed at the level of the aortic valve from a modified apical view. ${ }^{21}$

\section{Repeatability analysis}

Where possible CMR scans were repeated using the initial imaging geometry (scan-rescan repeatability) after 5-15 min. Repeatability of analysis was also assessed from a single set of images by the same (intra-) and a different (inter-) observer. Echocardiographic quantification of LVO was repeated where satisfactory views were obtained and where infants slept or were quietly awake throughout the echocardiogram. In all cases, observers were blinded to prior data when analysing flow volumes.

\section{Statistical analysis}

Bland-Altman analysis was used for assessment of repeatability and to compare methodologies. ${ }^{22}$ The mean bias and limits of agreement (LOA or 'repeatability coefficient', $1.96 \times \mathrm{SD}$ of differences) were calculated, as was repeatability index (RI, limits of agreement/mean of measures) to allow comparison of repeatability between different measures. ${ }^{23}$ Associations between continuous variables were assessed by simple linear regression. A probability value of $<0.05$ was considered significant. Demographic data are presented as median and range. Hemodynamic variables are presented as mean and SD, with the 2.5 th centile (equivalent to $2 \mathrm{SD}$ below the mean) taken as the lower limit of normal.

\section{RESULTS \\ Subjects}

In total 108 infants were studied. A range of imaging sequences were employed in each infant to optimise SSFP and PC imaging, such that all sequences were not attempted in all infants. Median birth weight was 1627 (580-4140) g, birth gestation 32 (25-42) weeks, 53 infants were male. At scan postnatal age was 11 (1-94) days, weight 1845 (5904600) $\mathrm{g}$ and gestation 34 (26-42) weeks. Seventy-four infants had been admitted to the neonatal unit, the remaining 34 received care on the postnatal ward. Twelve infants were receiving supplemental oxygen at the time of the scan; three 
infants were on continuous positive airway pressure (CPAP). No scan was complicated by significant desaturation, bradycardia or hypothermia.

\section{PC imaging - external validation}

Quantification of flow in an external phantom by optimised PC sequences was highly correlated with actual flow $\left(\mathrm{r}^{2}=0.995\right.$, figure 2).

\section{PC imaging - normal ranges and repeatability}

Optimised PC sequences were performed in 28 stable infants, with normal echocardiograms and proven ductal closure. Images of sufficient quality for quantification of LVO, SVC and DAo flow were obtained in all 28 infants, images of sufficient quality for quantification of RVO were obtained in 22 infants. The 28 infants had median birth weight 1856 (965-4140) g, birth gestation $33(28-41)$ weeks. At scan postnatal age was 10 (2-22) days, weight 2055 (1015-4140) g, gestation 34 (29-41) weeks. Seventeen infants had been admitted to the neonatal unit, 11 were term or near-term infants on the postnatal ward. One infant was receiving supplemental oxygen at the time of the scan; no infant was on CPAP.

Mean and SD of LVO, RVO, and SVC and DAo indexed to weight are shown in table 1. LVO $(p=0.005)$, RVO $(p=0.11)$,

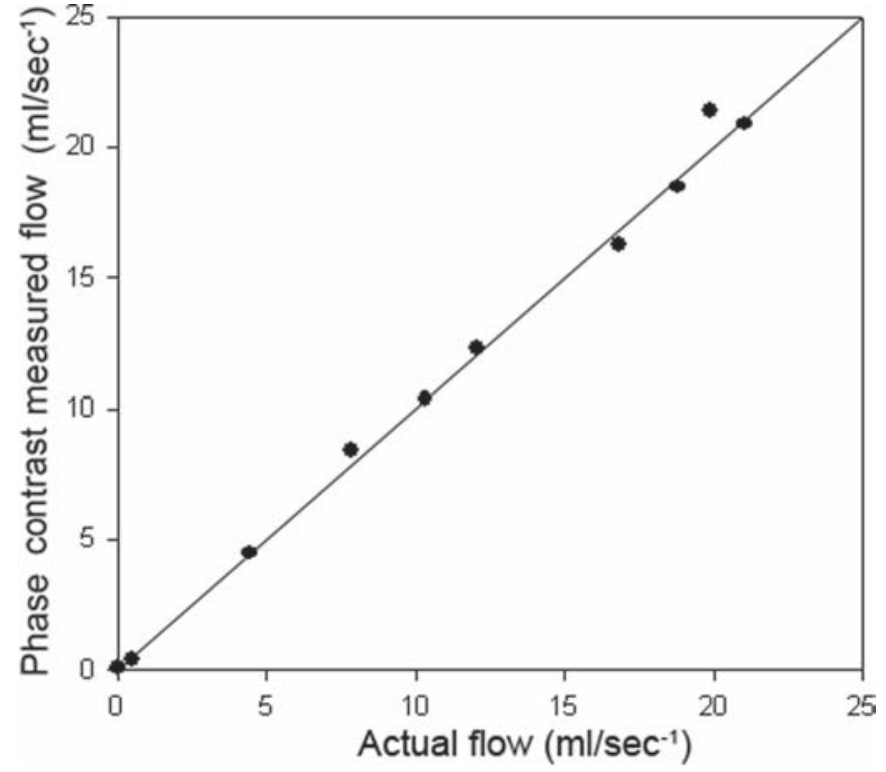

Figure 2 Regression plot of external validation of phase contrast flow quantification versus flow phantom. Line of unity shown.
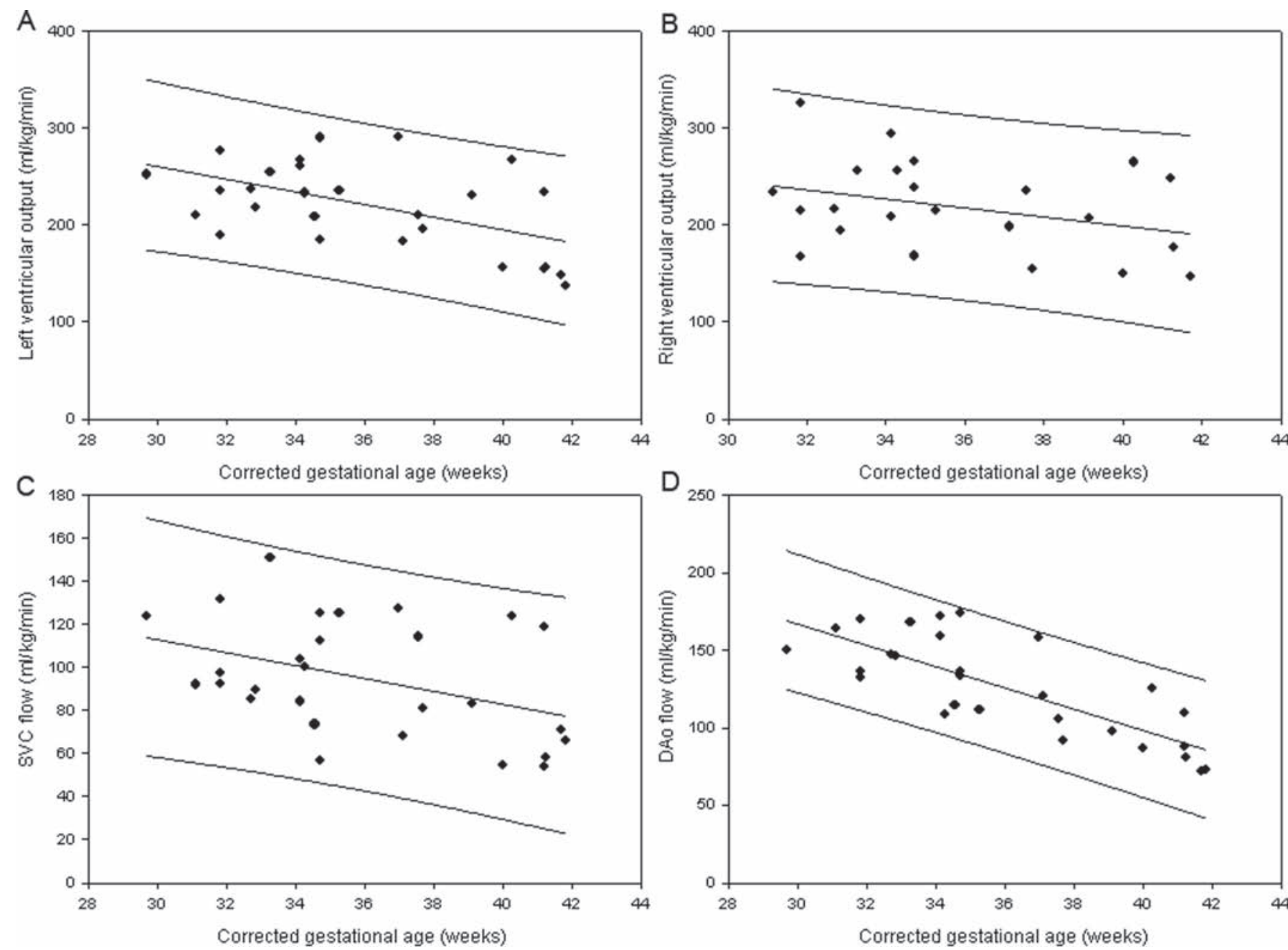

Figure 3 Normal ranges by corrected gestational age at scan for phase contrast assessment of left ventricular output (A), right ventricular output (B), superior vena caval flow (C) and descending aortic flow (D). 

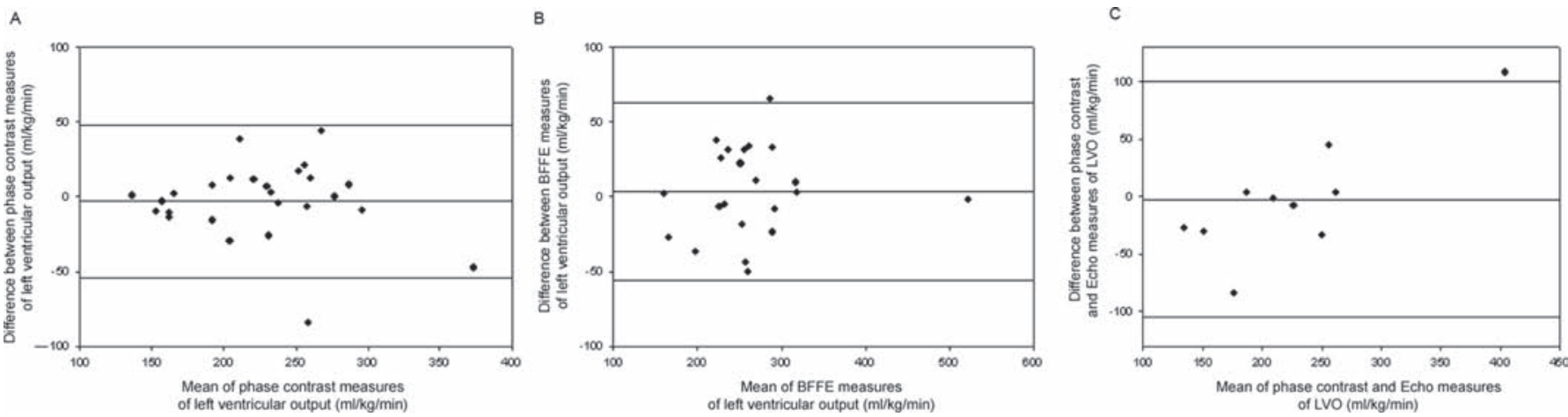

Figure 4 Bland-Altman plots of scan-rescan repeatability of assessment of left ventricular output performed by phase contrast (A) and cine (B) imaging, and of comparison of phase contrast and echo measures of left ventricular output (C).

Table 1 Phase contrast measures of left ventricular output (LVO), right ventricular output (RVO) and superior vena caval (SVC) and descending aortic (DAo) flow in 28 stable newborn infants

\begin{tabular}{llllll}
\hline & $\begin{array}{l}\text { LV0 } \\
(\mathbf{m l} / \mathbf{k g} / \mathbf{m i n})\end{array}$ & $\begin{array}{l}\text { RV0 } \\
(\mathbf{m l} / \mathbf{k g} / \mathbf{m i n})\end{array}$ & $\begin{array}{l}\text { SVC flow } \\
(\mathbf{m l} / \mathbf{k g} / \mathbf{m i n})\end{array}$ & $\begin{array}{l}\text { DAo flow } \\
(\mathbf{m l} / \mathbf{k g} / \mathbf{m i n})\end{array}$ & $\begin{array}{l}\text { SVC+DAo } \\
(\mathbf{m l} / \mathbf{k g} / \mathbf{m i n})\end{array}$ \\
\hline Mean & 222 & 219 & 95 & 126 & 224 \\
SD & 45.8 & 46.9 & 27.0 & 32.1 & 51.2 \\
\hline
\end{tabular}

Table 2 Repeatability of phase contrast measures of flow in newborn infants

\begin{tabular}{llcll}
\hline & LVO & RV0 & SVC & DA0 \\
\hline $\mathrm{n}$ & 26 & 9 & 24 & 21 \\
Limits of agreement $(\mathrm{ml} / \mathrm{kg} / \mathrm{min})$ & 50.2 & 55.5 & 20.9 & 26.2 \\
Repeatability index & $22.2 \%$ & $22.5 \%$ & $20.0 \%$ & $19.4 \%$ \\
\hline
\end{tabular}

SVC flow $(p=0.03)$ and DAo $(p<0.001)$ were lower at higher corrected gestational age (figure 3 ).

Table 2 shows the number of infants studied, LOA and RI for scan-rescan PC measures of LVO, RVO, and SVC and DAo flow indexed to weight within this group of 28 infants. Bland-Altman plot for scan-rescan repeatability of LVO by PC is shown in figure 4A. For most measures the scatter of the differences tends to increase as volume of flow increases, such that the LOA may be slightly wider than necessary for lower flows. Repeatability for LVO was similar when only preterm infants ( $<37$ weeks) were included $(\mathrm{n}=13, \mathrm{LAO} \pm 64.7 \mathrm{ml} / \mathrm{kg}$ / min, RI 25.1\%). Bland-Altman comparison of PC measures of combined systemic perfusion (SVC plus DAo flow) versus LVO in 30 infants, with echocardiographic exclusion of patent ductus arteriosus, showed mean difference $0.3 \mathrm{ml} / \mathrm{kg} / \mathrm{min}$, $\mathrm{LOA} \pm 46.7 \mathrm{ml} / \mathrm{kg} / \mathrm{min}, \mathrm{RI} 21.2 \%$. Repeatability of analysis of LVO from a single PC sequence in 10 infants showed intraobserver RI 8.2\%, inter-observer RI 8.3\%. Analysis duration was 2-3 min per vessel.

\section{Cine imaging - normal ranges and repeatability}

SSFP stacks with optimised parameters were acquired in 82 stable infants, without clinical evidence of patent ductus arteriosus. Thirty infants had proven ductal closure on echocardiogram and no infant subsequently received treatment for a patent ductus arteriosus during their NICU admission. Of these 82 infants, 75 had SSFP stacks of sufficient quality for analysis. These 75 infants had median birth weight 1886 (790-4140) g, birth gestation $33(25-42)$ weeks. At scan postnatal age was 9 (1-73) days, weight 2192 (790-4140) g, gestation 35 (28-42) weeks. Forty-six infants had been admitted to the neonatal unit, 29 were term or near-term infants on the postnatal ward. Six infants were receiving supplemental oxygen at the time of the scan; one infant was on CPAP.

SNR and CNR of the optimised SSFP images in 10 infants were $\mathrm{SNR}_{\text {blood }} 50.2, \mathrm{SNR}_{\text {myocardium }} 141.2 ; \mathrm{CNR}_{\text {blood-myocardium }}$ 91.0.

Left ventricular functional parameters are shown in table 3. LVO was significantly lower at higher corrected gestational age $(\mathrm{p}<0.001)$ (figure $5 \mathrm{~A})$. There was no significant association between gestational age and left ventricular EDV $(p=0.19)$, $\operatorname{ESV}(p=0.80)$ or EF $(p=0.18)$ (figure $5 B-D)$. The 2.5 th centiles for EDV, ESV and EF were $1.8 \mathrm{ml} / \mathrm{kg}, 0.4 \mathrm{ml} / \mathrm{kg}$ and $58 \%$, respectively.

Table 4 shows the LOA and RI for scan-rescan cine measures of left ventricular functional parameters within this group of 75 infants. Bland-Altman plot for scan-rescan repeatability of LVO by cine is shown in figure $4 \mathrm{~B}$. For most measures, the scatter of the differences tends to increase as volume increases such that again LOA may be wider than necessary for lower measures. Repeatability for assessment of LVO was similar when only preterm infants ( $<37$ weeks) were considered in the analysis $(\mathrm{n}=12, \mathrm{LOA} \pm 65.6 \mathrm{ml} / \mathrm{kg} / \mathrm{min}$, RI $22.8 \%$ ). Repeatability of analysis of LVO from a single set of bFFE images using CMR Tools in 10 infants showed intraobserver RI 9.5\%, inter-observer RI 22.4\%. Analysis duration was approx 10-15 min per ventricle. Repeatability of right ventricular function from short axis stack cine images was poor $(\mathrm{LOA} \pm 126.3 \mathrm{ml} / \mathrm{kg} / \mathrm{min}$, RI $51.7 \%)$.

\section{Comparison with echo}

Echocardiography was performed in a total of 45 subjects. Scan-rescan repeatability of LVO by echocardiography in 10 infants who remained asleep or quietly awake throughout the echocardiogram showed LOA $\pm 108.9 \mathrm{ml} / \mathrm{kg} / \mathrm{min}$, RI $49.2 \%$. Bland-Altman comparison of LVO when measured by PC and echo in 10 infants showed mean difference $2.4 \mathrm{ml} / \mathrm{kg} / \mathrm{min}$, $\mathrm{LOA}+98.0$ to $-102.8 \mathrm{ml} / \mathrm{kg} / \mathrm{min}$, RI $44.4 \%$, figure $4 \mathrm{C}$ ).

\section{DISCUSSION}

Our group has developed a system for safely obtaining MR images in preterm and term infants. ${ }^{16}$ Having previously demonstrated feasibility of performing basic CMR in newborns without sedation or anaesthesia, ${ }^{17}$ we now describe validation of optimised cine and PC techniques. High quality images demonstrating cardiac filling, cardiac output and systemic perfusion can be obtained in newborns. SNR and CNR of these images are equivalent to those obtained at $3 \mathrm{~T}$ in adult subjects. ${ }^{20}$ Quantitative measures appear to be more repeatable than echocardiographic assessments; and CMR 
measures appear robust when subjected to flow phantom validation. While we do not envisage CMR becoming a widespread tool in clinical practice, we believe that it could prove to be a useful research technique to study small numbers of newborn infants in specialist centres; providing insights into the pathophysiology of circulatory failure; acting as an outcome measure in clinical trials of inotropic intervention and so guiding clinical practice in the wider neonatal community.

Table 3 Left ventricular indices taken from stacks of cine images in 75 stable newborn infants

\begin{tabular}{llllccc}
\hline & $\begin{array}{l}\text { LV EDV } \\
(\mathbf{m l} / \mathbf{k g})\end{array}$ & $\begin{array}{l}\text { LV ESV } \\
(\mathbf{m l} / \mathbf{k g})\end{array}$ & $\begin{array}{l}\text { LV SV } \\
(\mathbf{m l} / \mathbf{k g})\end{array}$ & $\begin{array}{l}\text { LV EF } \\
(\%)\end{array}$ & $\begin{array}{l}\text { HR } \\
(\mathbf{b p m})\end{array}$ & $\begin{array}{l}\text { LV0 } \\
(\mathbf{m l} / \mathbf{k g} / \mathbf{m i n})\end{array}$ \\
\hline Mean & 2.51 & 0.78 & 1.73 & 69.0 & 142 & 245 \\
SD & 0.40 & 0.21 & 0.28 & 5.7 & 17.1 & 47.1 \\
\hline
\end{tabular}

$E D V$, end-diastolic volume; EF, ejection fraction; ESV, end-systolic volume; $H R$, heart rate; LV, left ventricle; LVO, left ventricular output; SV, stroke volume.

Table 4 Repeatability of left ventricular indices in 22 newborn infants

\begin{tabular}{llllll}
\hline & EDV & ESV & SV & EF & LVO \\
\hline Limits of agreement & $1.07 \mathrm{ml}$ & $0.48 \mathrm{ml}$ & $0.88 \mathrm{ml}$ & $7.4 \%$ & $58.3(\mathrm{ml} / \mathrm{kg} / \mathrm{min})$ \\
Repeatability index & $19.4 \%$ & $27.7 \%$ & $23.1 \%$ & $10.8 \%$ & $22.1 \%$ \\
\hline
\end{tabular}

EDV, end-diastolic volume; EF, ejection fraction; ESV, end-systolic volume; LVO, left ventricular output; SV, stroke volume.
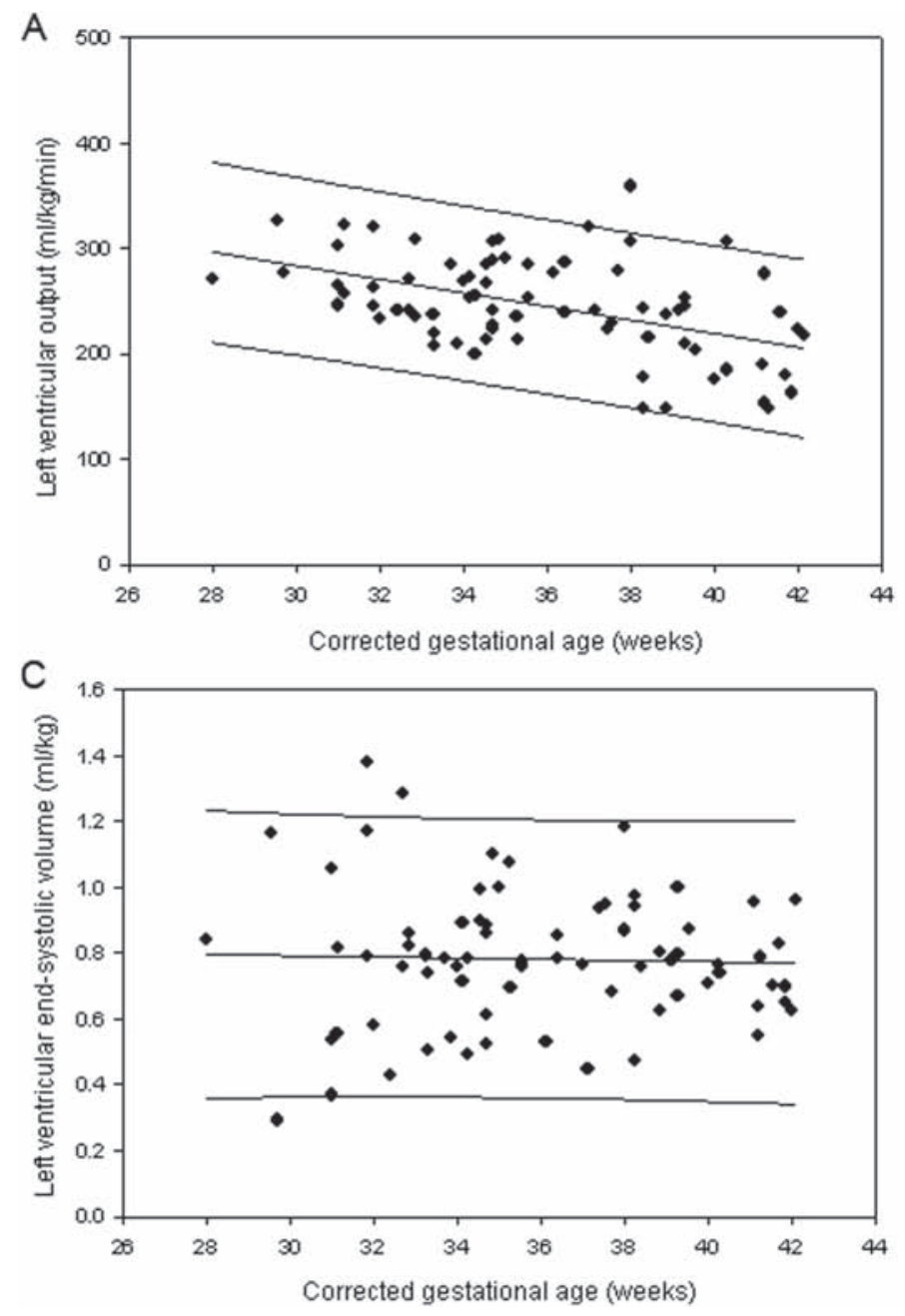

Current understanding of the neonatal circulation is heavily based on studies of blood pressure, which has a weak correlation with tissue perfusion. ${ }^{24}$ Intervention to increase blood pressure may not increase tissue perfusion ${ }^{25}$ or improve outcome. ${ }^{26}$ While echocardiography has value in providing functional circulatory assessments ${ }^{27}$ its accuracy is limited with studies suggesting that echo cannot reliably detect changes in neonatal cardiac output, ${ }^{9}$ or systemic perfusion ${ }^{10} 11$ of below 30-40\%, consistent with our findings of a RI of $49 \%$. Repeatability of echo measures may be improved if only changes in stroke distance are used. ${ }^{28}$ However, this approach does not assist with provision of absolute flow data; nor can it be used in vessels where diameter is variable such as the SVC. Novel circulatory biomarkers could improve understanding of the pathophysiology of neonatal circulatory failure and allow assessment of efficacy of inotropic intervention.

PC CMR is known to reliably quantify flow in adults. ${ }^{18} \mathrm{We}$ report optimised PC parameters for newborn infant imaging at $3 \mathrm{~T}$. A voxel size of $<1 \mathrm{~mm}$ and scan duration of $<60 \mathrm{~s}$ allow quantification of flow at multiple sites. A RI of $19-22 \%$ was consistently seen in preterm and term newborns. While this variability is higher than seen in adults, it is significantly lower than prior echocardiographic studies in newborns ${ }^{9-11}$ and will be partly due to the increase in spontaneous fluctuation of circulatory parameters seen in the newborn. ${ }^{11}$
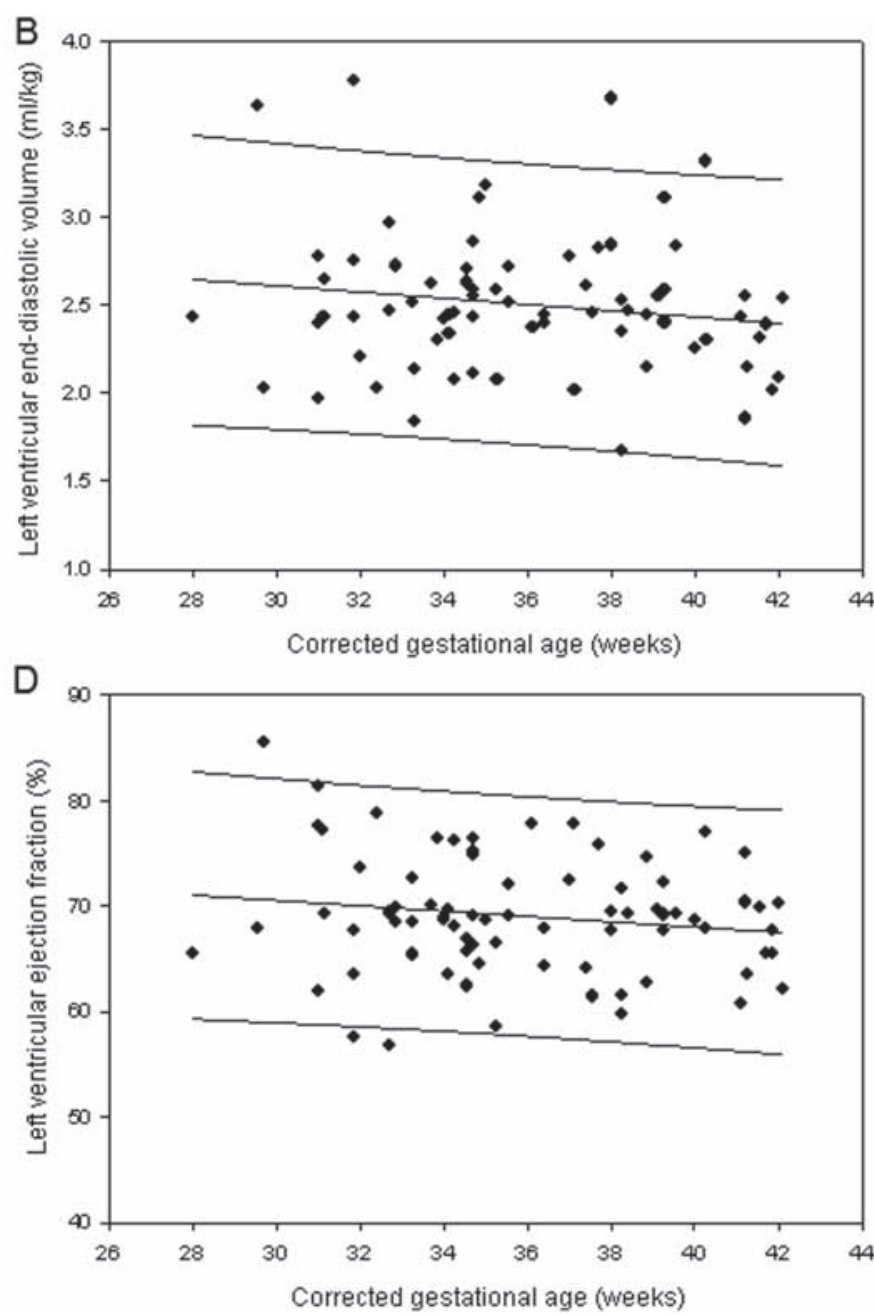

Figure 5 Normal ranges by corrected gestational age at scan for bFFE assessment of left ventricular output (A), end-diastolic volume (B), endsystolic volume (C) and ejection fraction (D). 
Quantification of ventricular volumes from stacks of cine images contain the entire ventricular volumes and do not rely on assumptions of ventricular geometry which are unreliable in the newborn. ${ }^{12}$ While such analysis must be performed offline, analysis duration was only 10-15 min, which is not prohibitive, particularly in the research setting. This analysis also provides repeatable and previously unavailable data on cardiac filling (EDV) and EF in the newborn.

The practical utility of CMR in preterm newborn infants is an important consideration. We have safely studied large number of infants. ${ }^{16}$ While CMR cannot replace echocardiography, it does provide additional value in range of data provided and in repeatability. As in adults this improved repeatability should translate into increased power for clinical trials. For example, to demonstrate a $20 \%$ increase in total systemic perfusion (SVC plus DAo, mean (SD) 224 (51) $\mathrm{ml} / \mathrm{kg} / \mathrm{min}$ ) between treated and control groups with $\alpha=0.05$ and $\beta=0.80$, requires just 16 infants per group. CMR could also increase insights into the pathophysiology of newborn circulatory failure and guide development of emerging echocardiographic techniques with greater applicability.

Our study has produced initial reference data for newborn circulatory function. Mean LVO, RVO, SVC flow and DAo flow as assessed by PC in stable infants were 222, 219, 95 and $126 \mathrm{ml} / \mathrm{kg} / \mathrm{min}$, respectively. The combined volume of SVC and DAo flow closely represents LVO when the ductus arteriosus is closed, suggesting that this could act as a surrogate for total systemic perfusion. Measures of flow tended to be higher at lower corrected gestational age, such that individual patients require gestation corrected normal ranges (figures 3 and $5 \mathrm{~A}$ ). Mean LVO as assessed by SSFP was $245 \mathrm{ml} / \mathrm{kg} / \mathrm{min}$, but again higher at lower corrected gestational age. Measures of left ventricular filling and ejection did not vary with gestational age, with EDV $<1.8 \mathrm{ml} / \mathrm{kg}, \mathrm{ESV}<0.4 \mathrm{ml} / \mathrm{kg}$ and $\mathrm{EF}<58 \%$ all likely to be abnormally low in the newborn.

This study has a number of limitations. The final optimised imaging parameters were not implemented in all infants since an initial protocol development phase was required for the techniques. Echocardiographic quantification of LVO was only introduced once the optimal cine and PC parameters were identified, and even then many infants were too unsettled for repeated echocardiographic measurements to be performed. Without echo imaging the presence of sub-clinical ductal shunting cannot be excluded in the cohort of infants studied by cine CMR. We have not yet studied large number of extremely preterm infants, though this was not considered a priority during the optimisation process. We have not yet implemented an alternative approach (such as axial imaging) for the assessment of right ventricular function, but an alternative to poorly repeatable short axis images will be required.

\section{CONCLUSIONS}

This study confirms the feasibility of CMR in newborn infants and demonstrates that $\mathrm{CMR}$ provides additional value over echocardiography. Initial gestation-related reference values for cardiac filling, cardiac output and systemic perfusion are provided. CMR has the potential to measure cardiovascular function in preterm infants with sufficient precision to allow appropriately powered trials of therapeutic intervention in fewer numbers of critically ill patients.

Acknowledgements The authors are grateful to the families who took part in the study, to the nursing and medical staff who cared for the infants, and to the staff of the Imaging Sciences Department of Imperial College London.
Funding This study was supported by the Garfield Weston Foundation, the Medical Research Council, the Wellcome Trust, the Imperial College Comprehensive Biomedical Research Centre and a Dorothy Hodgkins Postgraduate Award (S.U.).

Competing interests $\mathrm{RR}, \mathrm{JVH}$ and $\mathrm{ADE}$ receive investigator-led research support from Philips.

Ethics approval This study was conducted with the approval of the Hammersmith Hospital REC.

Provenance and peer review Not commissioned; externally peer reviewed.

\section{REFERENCES}

1. Mangham LJ, Petrou S, Doyle LW, et al. The cost of preterm birth throughout childhood in England and Wales. Pediatrics 2009;123:e312-27.

2. Doyle LW, Gultom E, Chuang SL, et al. Changing mortality and causes of death in infants 23-27 weeks' gestational age. J Paediatr Child Health 1999;35:255-9.

3. Volpe JJ. Neurobiology of periventricular leukomalacia in the premature infant. Pediatr Res 2001;50:553-62.

4. Parker TA, Abman SH. The pulmonary circulation in bronchopulmonary dysplasia. Semin Neonatol 2003;8:51-61.

5. Barrington KJ. Hypotension and shock in the preterm infant. Semin Fetal Neonatal Med 2008;13:16-23.

6. Whitsett JA, Noguchi A, Moore JJ. Developmental aspects of alpha- and betaadrenergic receptors. Semin Perinatol 1982;6:125-41.

7. Al-Aweel I, Pursley DM, Rubin LP, et al. Variations in prevalence of hypotension, hypertension, and vasopressor use in NICUs. J Perinatol 2001;21:272-8.

8. Jobe $\mathbf{A H}$. The cardiopulmonary system: research and training opportunities. J Perinatol 2006;26(Suppl 2):S5-7.

9. Chew MS, Poelaert J. Accuracy and repeatability of pediatric cardiac output measurement using Doppler: 20-year review of the literature. Intensive Care Med 2003;29:1889-94.

10. Kluckow M, Evans N. Superior vena cava flow in newborn infants: a novel marker of systemic blood flow. Arch Dis Child Fetal Neonatal Ed 2000;82:F182-7.

11. Groves AM, Kuschel CA, Knight DB, et al. Echocardiographic assessment of blood flow volume in the superior vena cava and descending aorta in the newborn infant. Arch Dis Child Fetal Neonatal Ed 2008;93:F24-8.

12. Lee LA, Kimball TR, Daniels SR, et al. Left ventricular mechanics in the preterm infant and their effect on the measurement of cardiac performance. J Pediatr 1992; 120:114-19.

13. Finn JP, Nael K, Deshpande V, et al. Cardiac MR imaging: state of the technology. Radiology 2006;241:338-54

14. Grothues F, Smith GC, Moon JC, et al. Comparison of interstudy reproducibility of cardiovascular magnetic resonance with two-dimensional echocardiography in normal subjects and in patients with heart failure or left ventricular hypertrophy. Am J Cardiol 2002;90:29-34.

15. Bellenger NG, Davies LC, Francis JM, et al. Reduction in sample size for studies of remodeling in heart failure by the use of cardiovascular magnetic resonance. $J$ Cardiovasc Magn Reson 2000;2:271-8.

16. Merchant $\mathbf{N}$, Groves A, Larkman DJ, et al. A patient care system for early 3.0 Tesla magnetic resonance imaging of very low birth weight infants. Early Hum Dev 2009;85:779-83.

17. Foran AM, Fitzpatrick JA, Allsop J, et al. Three-tesla cardiac magnetic resonance imaging for preterm infants. Pediatrics 2007;120:78-83.

18. Kilner PJ, Gatehouse PD, Firmin DN. Flow measurement by magnetic resonance: a unique asset worth optimising. J Cardiovasc Magn Reson 2007;9:723-8.

19. Walther FJ, Siassi B, King J, et al. Echocardiographic measurements in normal preterm and term neonates. Acta Paediatr Scand 1986;75:563-8.

20. Michaely HJ, Nael K, Schoenberg SO, et al. Analysis of cardiac functioncomparison between 1.5 Tesla and 3.0 Tesla cardiac cine magnetic resonance imaging: preliminary experience. Invest Radiol 2006;41:133-40.

21. Kluckow M, Evans N. Relationship between blood pressure and cardiac output in preterm infants requiring mechanical ventilation. J Pediatr 1996;129:506-12.

22. Bland JM, Altman DG. Statistical methods for assessing agreement between two methods of clinical measurement. Lancet 1986;1:307-10.

23. Bland JM, Altman DG. Applying the right statistics: analyses of measurement studies. Ultrasound Obstet Gynecol 2003;22:85-93.

24. Groves AM, Kuschel CA, Knight DB, et al. Relationship between blood pressure and blood flow in newborn preterm infants. Arch Dis Child Fetal Neonatal Ed 2008;93:F29-32.

25. Osborn D, Evans N, Kluckow M. Randomized trial of dobutamine versus dopamine in preterm infants with low systemic blood flow. J Pediatr 2002;140:183-91.

26. Batton B, Batton D, Riggs T. Blood pressure during the first 7 days in premature infants born at postmenstrual age 23 to 25 weeks. Am J Perinato/ 2007; 24:107-15.

27. Evans N. Echocardiography on neonatal intensive care units in Australia and New Zealand. J Paediatr Child Health 2000;36:169-71.

28. Tsai-Goodman B, Martin RP, Marlow N, et al. The repeatability of echocardiographic determination of right ventricular output in the newborn. Cardiol Young 2001;11:188-94. 